\title{
Current trends in mass spectrometry imaging
}

\author{
Andreas Römpp • Uwe Karst
}

Received: 8 January 2015 / Accepted: 9 January 2015 / Published online: 4 February 2015

(C) Springer-Verlag Berlin Heidelberg 2015

Mass spectrometry imaging provides spatial and molecular information for a wide range of analyte ions. The ability to map the distribution of biomolecules without labeling of target compounds has made imaging one of the most dynamic fields in mass spectrometry. In continuation of the ABC special issue "MALDI Imaging" in 2011 (Vol. 401, Issue 1), we cover recent trends in mass spectrometry imaging. The community has been very active and we have seen a number of exiting developments in instrumentation and applications. While MALDI is still the most widely used technique for mass spectrometry imaging, a number of alternative approaches, including ambient ionization sources, have been used increasingly in the last few years. In addition, sample preparation and data processing strategies have received increased interest in the MS imaging community. Sample preparation includes on-tissue chemistry, e.g., for digestion of proteins. This is related to a continuing shift away from imaging of (unidentified) intact proteins towards imaging of "metabolites" and drug compounds in the lower mass range. This was enabled by the increased use and availability of high resolution mass spectrometers, which allow differentiation of

Published in the topical collection Mass Spectrometry Imaging with guest editors Andreas Römpp and Uwe Karst.

\footnotetext{
A. Römpp $(\bowtie)$

Institute of Inorganic and Analytical Chemistry, Justus Liebig

University, Schubertstrasse 60, 35392 Giessen, Germany

e-mail: andreas.roempp@anorg.chemie.uni-giessen.de

U. Karst

Institute of Inorganic and Analytical Chemistry, University of

Münster, Corrensstraße 30, 48149 Muenster, Germany

e-mail: uk@uni-muenster.de
}

analyte signals from matrix background (without the need for MS/MS). In addition, a growing number of studies include complementary techniques such as LCMS/MS for the reliable identification of the imaged compounds. Another trend has been the use of multimodal approaches by employing multiple instrument platforms for mass spectrometry imaging and/or by integrating spectroscopic imaging techniques. The (absolute) quantitation of imaged compounds and the related issue of ion suppression have become one of the most discussed topics in the field. Finally, the rapidly increasing number of samples and size of data sets has boosted the development of strategies for analyzing and handling data. These general trends and developments in mass spectrometry imaging are also reflected in the contributions to this topical collection as discussed in the following.

A key parameter for MS imaging experiments is spatial resolution. A few years ago pixel sizes of $10 \mu \mathrm{m}$ and below had only been demonstrated by very few groups for mammalian tissue sections. Several approaches are now being employed in order to obtain more detailed spatial information from a more diverse of set of samples. Bhandari et al. present the application of "mass spectrometry imaging with high resolution in mass and space" for the localization of metabolites in whole insects and individual insect organs at $10 \mu \mathrm{m}$ pixel size. Lee et al. investigate metabolites in maize leaves with a pixel size of $5 \mu \mathrm{m}$ and high resolution MALDI-MS with a modified commercial system. Zavalin et al. have significantly increased measurement speed for their transmission geometry approach and present the analysis of intact proteins at a pixel size of $2.5 \mu \mathrm{m}$ in MALDI-ToF-MS. Appropriate sample preparation is a key requirement for any high spatial resolution imaging method. In this context Anderson et al. 
present an approach to reduce washing steps for the analysis of proteins. Oversampling is another interesting approach to improve spatial resolution, which is demonstrated by Nazari et al. for IR-MALDESI-MS. Secondary ion mass spectrometry (SIMS) inherently allows one to address higher spatial resolution down to $100 \mathrm{~nm}$, as shown by Draude et al. for freeze-fractured epithelial plasma membranes.

A number of chemical modifications of the (tissue) sample have been developed in order to make additional compounds accessible for detection or to specifically enhance certain compound classes. The most prominent example is on-tissue digestion of proteins. The experimental parameters for this approach are systematically investigated by Diehl et al. Alternative use of enzymes include the tissue-specific analysis of $\mathrm{N}$-glycosylation profiles, which is presented by Gustafsson et al. for formalin-fixed paraffin-embedded tissue. Another method of on-tissue chemistry is presented by Flinders et al. who apply hydrazine-based derivatization agents in order to selectively enhance the detection of carbonyl compounds.

Signal enhancement can also be obtained by reducing sample complexity, e.g., by transferring proteins selectively to functionalized surfaces prior to MALDI analysis (Fournaise et al.). Surface-enhanced detection of lipids in nanostructureassisted laser desorption/ionization (NALDI) without the use of matrix is discussed by Krasny et al.

Surface extraction and ambient desorption techniques have been established as powerful analytical tools which require little or no sample preparation. Many of these techniques are suitable for MS imaging, as shown by Laskin et al. for 3D imaging of lipids and metabolites in tissues using nano desorption electrospray (DESI)MS. Kertesz et al. successfully developed a dropletbased microextraction surface sampling system with ESI-MS/MS for spatially resolved analysis. Liquid extraction surface analysis (LESA)-MS with high mass resolution was combined by Menezes et al. with Raman microscopy to detect metabolites. Janfelt et al. investigated the distribution of the drug terfenadine and its metabolites in locusts by DESI-MS, thus proving that these techniques do also have valuable applications in pharmaceutical research.

The investigation of drug compound distribution and quantitation is one of the major application areas of MS imaging. Porta et al. present an overview on quantitative approaches in mass spectrometry imaging and present examples for cocaine and a tryptic peptide. Connell et al. investigate the relative increase of an anticancer drug in an in vivo animal model and compare it to an in vitro model (tissue in solution). Absolute quantitation of a drug compound by spotting standard solutions on tissue is presented by Buck et al. Another approach is to spike the drug compounds in tissue homogenate and this approach is now also described for lipids in mouse brain sections (Jadoul et al.). A uniform application of an internal standard was used for the analysis of an antiretroviral drug by IR-MALDESI (Bokhart et al.). Kuchar et al. apply mass spectrometric and immunohistochemical imaging techniques to study the effects of Fabry disease by the renal sphingolipid distribution in a mouse model and compare the respective data to ESIMS/MS

Rodent animal model tissue is the preferred sample for establishing and optimizing MS imaging methods. This also applies to most studies in this topical collection as the emphasis is on new methodology. An exception is presented by Dekker et al. who describe a strategy to determine the metabolic state of human tumor tissue based on MS imaging with high mass resolution. Cillero-Pastor et al. investigate differentially expressed proteins (after on-tissue digestion) in the pathology of osteoarthritis by employing PCA.

While most applications of MS imaging are associated with biological questions arising at the tissue or cellular level, current techniques may also cover exciting new instrumentation and applications with relevance to environmental chemistry and/or human exposure monitoring: Hertz-Schünemann et al. propose microprobe sampling-photoionization mass spectrometry for high resolution temporal and spatial distribution mapping of several tobacco combustion and pyrolysis products in cigarette puff. Syed et al. describe the development of a micro pixelated ion imaging detector for mass resolution improvement of a quadrupole instrument.

No matter which approach is used, data processing is an integral part of any MS imaging experiment. An approach to speed up image generation for large data sets by use of distributed computing resources is described by Smith et al. A systematic study of different methods for denoising of images with a focus on low intensity signals is presented by Schwartz et al.

In addition to the methodological developments outlined above, the last few years have seen a number of collaborative efforts towards standardization and consolidation of MS imaging. An activity that targets these issues is the COST Action (European Cooperation in Science and Technology) "Mass spectrometry imaging: new tools for healthcare research" (BM1104) [1] which includes researchers from 23 European countries. Another 
example is the common data format imzML [2], which is directly supported by major instrument vendors as well as by open source software and has thus become a de facto standard format for MS imaging data. A feature article presents the use of imzML for implementation of a public data repository and the first 'open data' example for mass spectrometry imaging (Römpp, Wang, et al.). Another step towards more transparent and reproducible presentation of MS imaging data is reporting guidelines which are proposed in a second feature article (McDonnell et al.). The impact of color scales on the visualization of MS images is discussed in a critical review by Race et al. An important task for future MS imaging studies is the comparison and combination of methods and instrumentation across different laboratories. An approach on how to conduct such 'multicenter studies' is discussed in a note by Römpp, Both, et al. We believe that the combination of these collaborative activities will aid in establishing MS imaging as a proven and reliable technique in biomedical and bioanalytical applications in the future. We would like to thank all authors and reviewers who have contributed to this topical collection "Mass Spectrometry Imaging".

\section{References}

1. McDonnell LA, Heeren RMA, Andrén PE, Stoeckli M, Corthals GL (2012) Going forward: increasing the accessibility of imaging mass spectrometry. J Proteomics 75(16):5113-5121

2. Schramm T, Hester A, Klinkert I, Both J-P, Heeren RMA, Brunelle A, Laprévote O, Desbenoit N, Robbe M-F, Stoeckli M, Spengler B, Römpp A (2012) imzML - a common data format for the flexible exchange and processing of mass spectrometry imaging data. $\mathrm{J}$ Proteomics 75(16):5106-5110

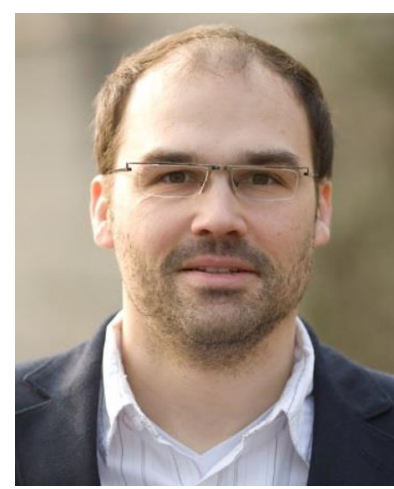

Andreas Römpp is a group leader and lecturer ("Privatdozent") at the Institute of Inorganic and Analytical Chemistry of Justus Liebig University in Giessen, Germany. He worked as a research assistant at the Max Planck Institute for Chemistry and obtained his $\mathrm{PhD}$ from the University of Mainz in 2003. In 2004 he worked as a postdoctoral researcher at the FOM-AMOLF Institute in Amsterdam. His work has always been focused on method development for high resolution mass spectrometry. In recent years mass spectrometry imaging has become his main area of interest with a particular focus on increasing spatial resolution and obtaining reliable chemical information. Andreas Römpp is coordinator of the common data format for mass spectrometry imaging-imzML. He is also actively involved in the MS imaging COST Action BM1104 as management committee member and work group co-chair.

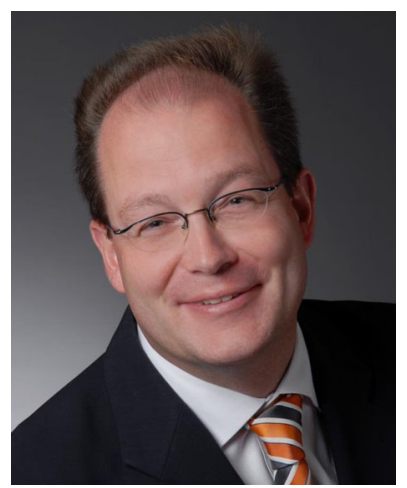

Uwe Karst obtained his Ph.D. in analytical chemistry from the University of Münster, Germany, in 1993, and was a Postdoctoral Research Associate at the University of Colorado in Boulder, CO, USA. After his return to the University of Münster, he finished his Habilitation in 1998. In 2001, he took over a position as Full Professor of Chemical Analysis at the University of Twente, the Netherlands. He accepted his current position as Chair of Analytical Chemistry at the University of Münster in 2005. His research interests focus on hyphenated analytical techniques and their (bio)medical and pharmaceutical applications, including elemental speciation analysis, metallomics, mass spectrometric imaging, and electrochemistry/MS. 